\title{
4. Prognosis of Patients with Rheumatic Heart Disease and Some Aspects on Its Prevention
}

\author{
Hironori Toshima, M. D. \\ The Third Department of Medicine (Director, Prof. N. Kimura), \\ Kurume University Medical School
}

It has recently been reported that the incidence of rheumatic heart diseases (RHD) at school age in Japan is about one tenth of that of congenital heart diseases, namely $0.03 \%$ and $0.3 \%$ respectively. ${ }^{1222}$

Incidence of RHD in the adult is not clear yet, but RHD is still one of the major causes of deaths and socioeconomical problem in Japan. Since 1965, prosthetic valve replacements have been carried out in 66 cases with RHD at Kurume University Hospital. However, the long term follow-up study in the comparison with the natural history has not been obtained.

Four hundreds and fifty-five cases with RHD admitted to our clinic during 1962 to 1971 were analysed. One hundred and fifty-four cases out of 455 were followed after their discharge without operation, being 103 cases still alive during the observation period, ranging from 1.0 to 11.0 years (average 4.9 years), and 51 cases died at 2.8 years ( 0.2 to 7.5$)$ after discharge. Three hundreds and one cases were subjected to cardiac surgery; 235 cases of mitral commissurotomy (MC), 46 cases of mitral valve replacement (MVR), and 20 cases of aortic valve replacement (AVR). 260 cases were still alive for 6.0 years $(1.0$ to 10.9) of observation period and 41 cases

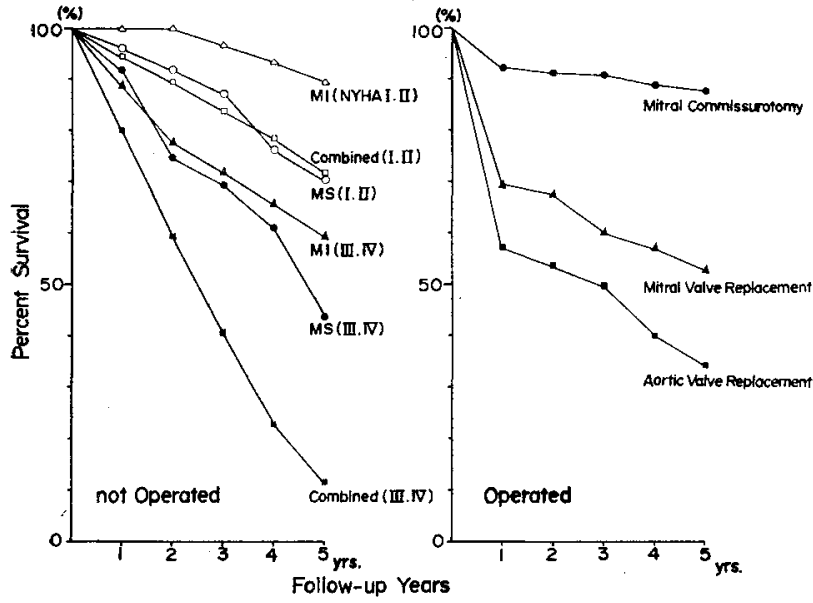

Fig. 1. Survival rate in patients with RHD. died at 2.9 years $(0.2$ to 8.0$)$.

Fig. 1 shows survival rate in patients with RHD during 5 years after discharge. Patients with combined valvular disease having functional classification of class III and class IV showed the most poor survival rate, that is, $11.3 \%$ at 5 years of follow-up. Increase in survival rates was seen in order of mitral stenosis with class III and IV, mitral insufficiency with class III and IV, mitral stenosis with class I and II, combined lesion with class I 
and II and mitral insufficiency with class I and II. While, the survival rate in the patients with MC was more excellent than that in the patients without operation and those with prosthetic valve replacement. These results would indicate that the survival rate of $\mathrm{MC}$ is absolutely better than that of natural history, but the survival rate of prosthetic valve replacement seems to be not always superior to the natural survival.

Significant risk factors in relation to death after discharge without operation in patients with mitral insufficiency were the history of congestive heart failure $(\mathrm{p}<0.005)$, cardiothoracic ratio more than $60 \%(\mathrm{P}<0.005)$, pulmonary systolic pressure more than $60 \mathrm{mmHg}(\mathrm{P}<0.01)$ and cardiac index less than $2.5 \mathrm{~L} / \mathrm{min} / \mathrm{m}^{2}(\mathrm{p}<0.025)$. While, there were no significant differences in the above-mentioned risk factors in relation to the operative and late death after MVR. Late deaths after prosthetic valve replacement were caused mostly by congestive heart failure due to the thrombosis of valve or cerebral embolism.

Thus, surgical treatment for RHD should be recommended to the cases having poor physical capacity or risk factors and medical treatment would be recommended to mild cases.

In 1961 an interesting case was referred to us because of heart murmur and diagnosed as RHD with mitral insufficiency by the author. In 1973 at his age of 36 years old, he complained of palpitation on exertion and visited our clinic again. At this time he was diagnosed as pure mitral stenosis. Change of the heart murmur after rheumatic fever has been reported by many investigators. ${ }^{3 / 4)}$ However, it has been very rare in adult that pure mitral insufficiency developed to pure mitral stenosis during 12 years after the first attack. Another interest is that this patient has had no symptoms suggesting rheumatic fever during this 12 years.

History of rheumatic fever was shown in only $22.8 \%$ of 92 cases with mitral stenosis and about $50 \%$ of 93 cases with other type of RHD. It is well known that rheumatic fever and RHD are preventable. The problem to be solved is that the patients with RHD manifested by rheumatic fever are not few. Namely, secondary prevention entails identifying patients who have RHD before development of subjective symptoms and administering adequate prophylaxis to prevent further streptococcal infections and subsequent attacks of rheumatic fever.

In Japan the invasion of pulmonary tuberculosis has been overwhelmed after the Ind World War. Such experience and system should be recognized and applied in order to identify the patients with RHD at their early stage of the disease. Mass screening examinations are widely and routinely performed at industrial groups, schools or other many groups, aiming initialiy to find pulmonary tuberculosis, recently to make early detection of cancer, hypertension or ischemic heart diseases especially for the people more than 40 years old. These efforts should include the people less than 40 years old to find out RHD at symptomless stage. Such procedure will lead to early prophylaxis. It is confirmed that the application of bipolar electrodes, $C_{5}-C_{5} R$, and phonocardiograms recorded with $M_{1}$ or $M_{2}$ filter at the apex and the base are 
useful to find out such subjects. It needs only a few minutes to perform these procedures. Expense for this additional examination equivalent to the cost for a single prosthetic valve replacement will cover at least ten thousand subjects. The cost for one operation of prosthetic valve replacement needs about 2 million yen.

\section{References}

1) Takayasu, M. et al.: Committee Reports, unpublished data.

2) Kawakita, S.: Jap. J. Clin. Med., $28: 1643,1970$.

3) Honma, M. and Tsunata, J.: Jap. J. Clin. Med., 28 : 1683, 1970.

4) Bland, E. F. and Jones, T. D. : Circulation, 4:836, 1951. 